\title{
Analysis of Splice Loss of Single-Mode Optical Fiber in the High Altitude Environment
}

\author{
Liwen $\mathrm{Hu} *$ (D) and Chaowei Yuan \\ School of Information and Communication Engineering, Beijing University of Posts and Telecommunications, \\ No.10, Xitucheng Road, Beijing 100876, China; yuancw2000@bupt.edu.cn \\ * Correspondence: lwhu214@bupt.edu.cn
}

Citation: Hu, L.; Yuan, C. Analysis of Splice Loss of Single-Mode Optical Fiber in the High Altitude Environment. Coatings 2021, 11, 876. https://doi.org/ 10.3390/coatings11080876

Academic Editor: Zhenyang Ding

Received: 19 June 2021

Accepted: 20 July 2021

Published: 22 July 2021

Publisher's Note: MDPI stays neutral with regard to jurisdictional claims in published maps and institutional affiliations.

Copyright: (c) 2021 by the authors. Licensee MDPI, Basel, Switzerland. This article is an open access article distributed under the terms and conditions of the Creative Commons Attribution (CC BY) license (https:// creativecommons.org/licenses/by/ $4.0 /)$.

\begin{abstract}
Up to now, there have been no complete theoretical researches and field experiment reports on the fiber fusion loss at high altitude. Therefore, we have conducted an exploratory study on the fiber splicing loss at high altitude, and firstly analyze the influence of mode field diameter mismatch, axial offset, angle tilt or end face gap affected by high altitude on splice loss, and then discuss the influence of fusion-splicing parameters on splice loss. Besides, a mathematical model for reducing the splicing loss of single-mode fiber at high altitude is established by combining the effects of temperature, humidity, oxygen content, atmospheric pressure, gale and gravity. We have conducted repeated field fusion experiments in different altitude areas (53, 2980, 4000, 4200, 4300, 5020, and $5200 \mathrm{~m}$ ) more than once, hence obtaining a large number of field experimental data, making a deep comparison between typical "plain" area and typical "high altitude" area. The splice loss of most fusion points achieved successfully has been reduced by at least $0.07 \mathrm{~dB}$. The simulation results are basically consistent with the theoretical analysis. Ultimately, the method proposed has been directly applied to on-site splicing engineering in high altitude environment and achieves good results.
\end{abstract}

Keywords: fusion splice; splice loss; high altitude environment; field experiment

\section{Introduction}

In the optical fiber communication links with a large span and super long distance, the whole optical fiber link is often composed of several independent and continuous fiber splicing [1,2]. However, the total loss stemming from the addition of joint losses does account for a large proportion of the whole link loss [1,3]. Besides, splicing joints also often exist in optical devices [3]. Fortunately, how to reduce the fiber splice loss has been widely concerned and studied in plain areas [2-14]. By effectively controlling the viscous flow or the diffusion of glass components in the fusion-splicing process, the loss caused only by lateral shift and refractive index change can be reduced [4]. However, this literature not only fails to consider the effect of angle tilt on the fusion splice loss, but also lacks enough experimental data. In addition, in literature [2,8-11], the optimal parameter combinations to minimize the splice loss have been given respectively under different conditions, such as $8.5 \mathrm{~W}$ discharge power and $2000{ }^{\circ} \mathrm{C}$ heating temperature [8], but the influence of mode field diameter mismatch and end face spacing on splice loss is not considered in this literature. The fusion loss of single-mode fiber with core diameters of 10 and $7 \mu \mathrm{m}$ is 0.07 and $0.15 \mathrm{~dB}$ respectively [9]. However, this literature does not consider the influence of mode field diameter mismatch on the splice loss, nor does it consider the gravity effect in the fusion-splicing process. When the eccentricity of the fiber core and the deviation of the outer diameter of the fiber exist, the change of splice loss can be less than $0.1 \mathrm{~dB}$ with the $18 \mathrm{~mA}$ discharge current, $0.2 \mathrm{~s}$ pre-fusion time and $1 \mathrm{~s}$ discharge duration [11], but the influence of angle tilt and end face spacing on splice loss is not considered here. In particular, these research results are all realized in plain areas. When we first apply the optimal fusion methods given by these research results directly to the optical fiber 
fusion in high altitude area, the expected results are not obtained, even far from the field measurement results.

Though artificial operation, instrumentation and external environment have a great impact on the actual fusion process [3-7], there have been no complete theoretical researches and field experimental reports on fiber fusion at high altitude. Fortunately, the altitude can already be measured with great accuracy $[15,16]$. In addition to axial offset and angle tilt of the fiber $[9,11,13]$, mode field diameter mismatch and end face gap of the fiber can also have a great impact on the splice loss [17-20], so do the fusion-splicing device and parameters in the fusion process [21-23]. In recent years, some researchers have carried out statistical modeling and analysis on splice loss $[24,25]$. Therefrom, our research team has conducted exploratory research on splice loss under the special environment of high altitude for the first time. Moreover, another researcher in our laboratory has considered the effect of angle tilt on splice loss in literature [26], involving temperature and humidity. However, this literature does not research the influence of mode field diameter mismatch, axial offset and end face spacing on the splice loss, nor does it consider the gravity, oxygen content and wind in high altitude environment. Besides, the experimental data needed on in-situ fiber splicing at high altitude are seriously insufficient. Furthermore, the theory proposed does not achieve the expected theoretical estimate of splice loss when fusion-splicing in situ is implemented at high altitude.

Therefore, this paper continues to conduct exploratory research on fiber fusion loss in high altitude environment. We firstly analyze the influence of mode field diameter mismatch, axial offset, angle tilt or end face gap affected by high altitude on splice loss, and then discuss the influence of fusion parameters on splice loss. Though the optimal fusion combination conditions given in the published literature are directly applied to the exploratory experiment of in-situ splicing in high altitude area for the first time, not only is it difficult to splice successfully, but also most of the measured loss values are more than $0.5 \mathrm{~dB}$, which greatly exceeds the loss budget. To tackle this problem, a mathematical model for reducing the splice loss at high altitude is established, involving temperature, humidity, oxygen content, atmospheric pressure, gale and gravity. Moreover, the main causes of high splice loss in high altitude environment are analyzed and identified. Then, we once again carry out several repeated field fusion experiments at different altitudes, making a deep comparison between typical "plain" area (53 m) and typical "high altitude" area $(4300 \mathrm{~m})$, hence obtaining a large number of field experimental data. Most of the splice loss measurement values are less than $0.5 \mathrm{~dB}$. More importantly, some of the splice loss is even reduced to about $0.3 \mathrm{~dB}$. In addition, the optimal combination conditions to minimize the splice loss of two different single-mode optical fibers in the high altitude environment are compared again. The simulation results are basically consistent with the theoretical analysis. Subsequently, the method proposed has been directly applied to the fiber splicing engineering in the high altitude environment, achieving good results, thus significantly reducing the cost of constructing a long-span and ultra-long-distance optical fiber communication link at high altitude, which has certain reference significance for the future research of splicing loss in the high altitude environment.

\section{Materials and Methods}

A large number of fusion platforms can successfully realize the fusion-splicing of two optical fibers [2,3,8-12], but due to the special harsh environment at high altitude, the experimental personnel can not stay for a long time. To solve this problem, this paper borrows the fusion-splicing platform proposed by A.K. Das [9,10]. This platform is easy to be built and operated, which is very conducive to the manual fusion-splicing operation in the high altitude environment, as shown in the simplified diagram at the lower right corner of Figure 1. In order to highlight the research focus of this paper, only the details of the butt-joint of the optical fiber ends in the fusion-splicing process are described in depth here. In particular, the optical fiber end face preparation technology commonly used $[3,8]$ is adopted, and the optical fiber end face obtained is assumed to be very ideal. 
After the preparation stage, the Sumitomo Type-81C fusion splicer (Sumitomo, Tokyo, Japan) is selected to complete the optical fiber fusion experiment, whose discharge power is AC discharge mode with the $100 \sim 240 \mathrm{~V}$ at $50 \sim 60 \mathrm{~Hz}$ discharge voltage, and the splicing set-up constructed by which is shown in the simple figure in Figure 1. Besides, the default parameters of this fusion splicer are: the tungsten electrode diameter is about $1 \mathrm{~mm}$; the gap distance between the electrodes is $2 \mathrm{~mm}$; the tapering angle of the electrode is $30^{\circ}$ under the standard atmospheric pressure. It is worth noting that this splicing set-up covers almost all operations in the fusion-splicing process, such as optical fiber preparation, alignment, joint formation, camera, splice packaging. Noteworthily, the fusion splicer is placed on the uneven surfaces, which can increase the difficulty of optical fiber alignment, such as increasing the axial offset and angle tilt. In addition, the oblique placement of the fusion splicer can also lead to serious uneven forces in the splicing process of the two optical fibers.

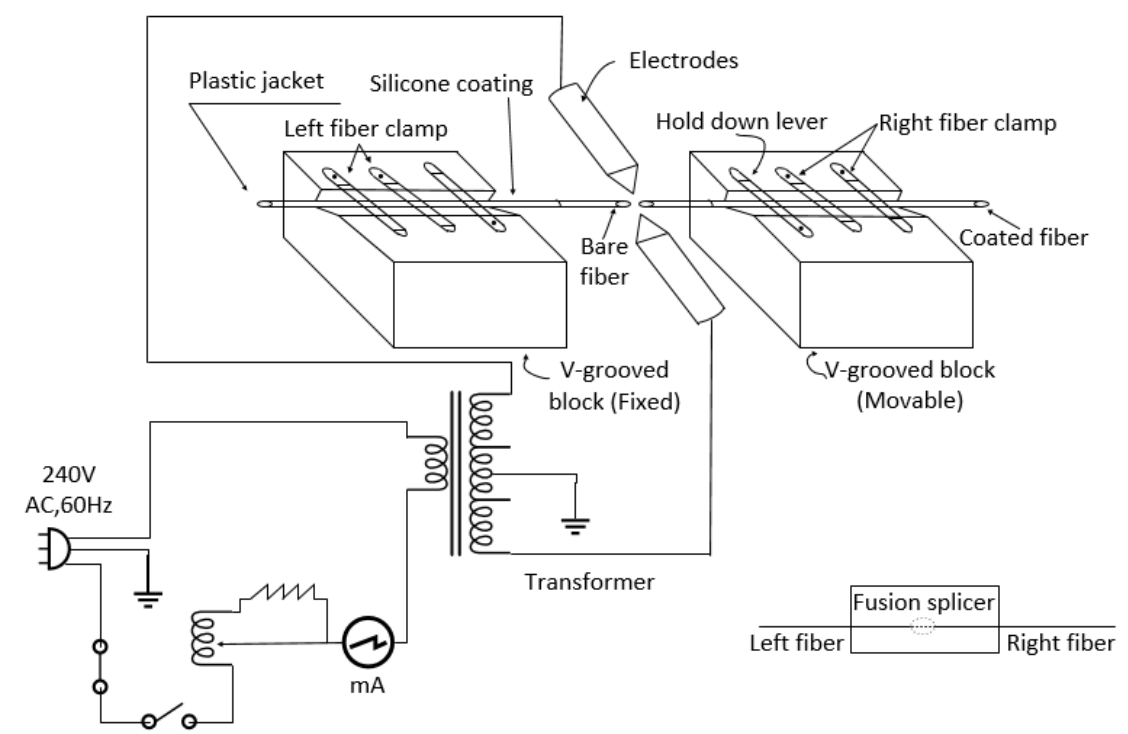

Figure 1. Splicing set-up, at high altitudes, the fusion splicer is placed on as flat a surface as possible to minimize unnecessary interference, as shown in the lower right corner of the figure. The left and right optical fibers are respectively placed in the V-groove of the fusion splicer, being aligned with each other. Then, the fusion splicer quickly discharges and heats the fiber ends through two electrodes, which makes it fuse and splice together quickly.

In the fusion-splicing process, the electrode discharges to heat the ends of two optical fibers, meanwhile, the optical fiber at the moving end is constantly moved to the fixed optical fiber under the force exerted by the pushing arm and the spring, so that the two optical fibers are squeezed together by the force at the same time, as shown in the dotted line in Figure 2a. In order to research the influence of high altitude environment on optical fiber fusion-splicing more accurately, this paper assumes that the power of optical fiber cladding mode is perfectly eliminated.

The main factors causing the fusion-splicing loss are the mismatch of the mode field diameter (MFD) of the optical fiber, the lateral offset of the optical fiber, the angle tilt of the optical fiber or the end face gap of the optical fiber $[3,13]$. The detailed characteristics of these four cases are shown in Figure 3. It is worth noting that literature [26] has neither researched the MFD mismatch, axial offset and end face gap, nor researched the gravity and heat flow under the fusion process in implementing the specific analysis. 


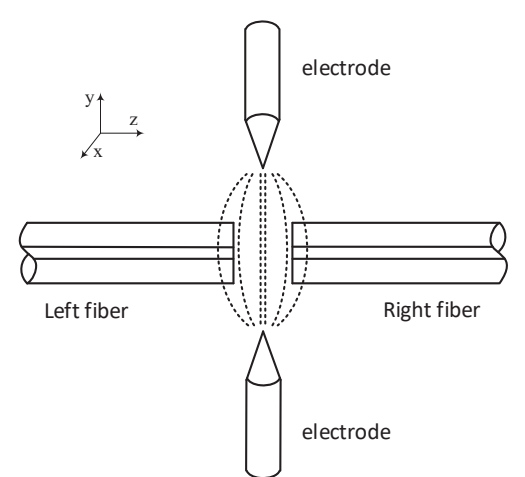

(a)

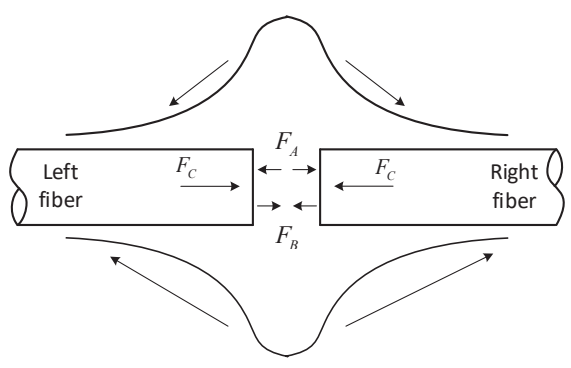

(b)

Figure 2. Schematic diagram of single-mode fiber fusion-splicing, (a): optical fiber fusion splice, the axis direction of the optical fiber is located in the Z-axis direction, and dotted line shows the two electrodes discharge and heat the two optical fibers simultaneously; (b): thermal and mechanical properties of the process of fusion-splicing; $F_{A}$ represents the surface tension of the end face of the optical fiber; $F_{B}$ represents the force exerted by the fusion splicer; $F_{C}$ represents the thermal expansion force; the gravity $F_{g}$ is not drawn here, and the heat source is transmitted along the axial direction of the two optical fibers and gradually weakens.

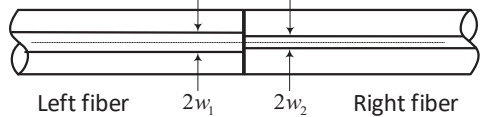

(a)

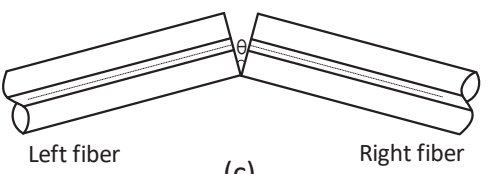

(c)

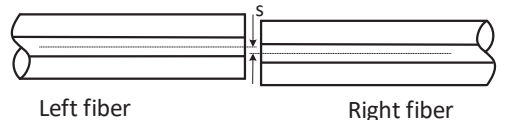

(b)

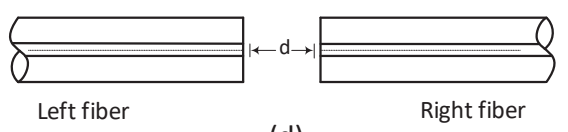

(d)

Figure 3. The main factors of fusion-splicing loss, (a): MFD mismatch, the mode field diameters of the left and right fibers are $2 w_{1}$ and $2 w_{2}(\mu \mathrm{m})$ respectively; $(\mathbf{b})$ : lateral offset, the two optical fiber end faces are laterally offset by $s(\mu \mathrm{m})$; (c): angle tilt, the end faces of the two fibers are tilted by $\theta$ degrees; (d): end face gap, the end faces of the two optical fibers are $d(\mu \mathrm{m})$ apart.

\subsection{Coupling Efficiency}

As can be seen from Figure $3 a$, the core diameter $2 r_{1}$ of the left fiber is significantly larger than the core diameter $2 r_{2}$ of the right fiber. According to the optical fiber propagation theory [1], when the optical signal propagates from the right optical fiber to the left optical fiber, it can be transmitted intact and lossless. Propagation from left to right can lead to a large attenuation. This is attributed to the coupling efficiency $\tau$ at the fusion joint of two optical fibers, namely, the optical power $P_{r}$ transmitted by the fundamental mode waveguide of the receiving end fiber dividing by the optical power $P_{t}$ transmitted by the fundamental mode waveguide of the transmitting end fiber [14]. The loss $L$ of the fusion joint can be expressed by the coupling efficiency $\tau$, namely:

$$
L=-10 \log \tau
$$

Obviously, from Equation (1), as long as the coupling efficiency $\tau$ of the fusion joint is calculated, the loss $L$ of the fusion joint can be obtained.

The mismatch of MFD has a great influence on splice loss [17,19]. The mode field distribution of step type single-mode fiber is very similar to Gaussian shape $[13,14]$. The spatial coordinate system established is shown in Figure 2a. There are five typical definitions of MFD in common use. In view of the characteristics of step type single-mode fiber, this paper uses the famous definition of MFD, namely Petermann II spot size, to describe 
the mode field diameter whose size is $2 w$. In addition, the value of $w$ can be obtained from the empirical formula [13].

$$
\frac{w}{r} \approx 0.65+\frac{1.619}{V^{3 / 2}}+\frac{2.879}{V^{6}}
$$

where $r$ is the core radius; $n_{1}$ and $n_{2}$ are the refractive index of the core and cladding respectively; $V=2 \pi r \sqrt{n_{1}^{2}-n_{2}^{2}} / \lambda$ is the normalized frequency, and here $0 \leq V \leq 2.5$.

It is assumed that the core eccentricity of both single-mode optical fibers can be neglected. According to the single-mode optical fiber fusion-splicing theory [13,14], when the loss caused only by (a) in Figure 3 occurs, that is, when two different types of optical fibers are fused successfully under ideal conditions without lateral offset, angular inclination or end face gap, the coupling efficiency $\tau$ of the fusion can be expressed as:

$$
\tau=\left(\frac{2 w_{1} w_{2}}{w_{1}^{2}+w_{2}^{2}}\right)^{2}
$$

Axial offset has a great influence on splice loss $[13,25]$. When the loss caused by $(b)$ in Figure 3 occurs, that is, when only transverse excursion occurs between two optical fibers, at this moment, the offset distance is $s$, without angular inclination and with the fine end face contact, so the coupling efficiency $\tau$ of the fusion can be expressed as:

$$
\tau=\left(\frac{2 w_{1} w_{2}}{w_{1}^{2}+w_{2}^{2}}\right)^{2} \exp \left(-\frac{2 s^{2}}{w_{1}^{2}+w_{2}^{2}}\right)
$$

The influence of angle tilt on splice loss is very large $[9,26]$. When the loss caused by (c) in Figure 3 occurs, that is, when only angular inclination occurs between two optical fibers, at this moment, the inclination angle is $\theta$, without transverse offset and with the fine end face contact, so the coupling efficiency $\tau$ of the fusion can be expressed as:

$$
\tau=\left(\frac{2 w_{1} w_{2}}{w_{1}^{2}+w_{2}^{2}}\right)^{2} \exp \left[-\frac{2 \pi^{2} n_{i}^{2} w_{1}^{2} w_{2}^{2} \sin ^{2} \theta}{\lambda^{2}\left(w_{1}^{2}+w_{2}^{2}\right)}\right]
$$

where $n_{i}$ is the refractive index of the uniform medium filled between two optical fibers, which can be air or immersion, etc.; $\lambda$ is the vacuum wavelength of the uniform plane wave with the same frequency.

The effect of end face gap on splice loss is also very huge $[3,18,20]$. When the loss caused by (d) in Figure 3 occurs, that is, when there is only an interval of $d$ between two optical fibers without lateral deviation or angular tilting. Besides, the Fresnel reflection loss is ignored here, so the coupling efficiency $\tau$ of the fusion can be expressed as:

$$
\tau=\frac{4 w_{1}^{2} w_{2}^{2}}{\left(w_{1}^{2}+w_{2}^{2}\right)^{2}+\left(\frac{\lambda d}{\pi n_{i}}\right)^{2}}
$$

\subsection{Environmental Impact of the High Altitude}

Figure 2a shows that the two optical fibers placed in the fusion splicer are heated by the discharge of the two electrodes to reach the corresponding softening temperature, thus fused together. During the whole process, the central position of the fusion region between the two optical fibers is most affected by the heat source, and then gradually weakens along the axial direction of the corresponding optical fiber core, as shown in the direction of the parabola and arrow in Figure 2b. Especially, the whole fusion-splicing process can be affected by the environment. According to the theory of optical fiber fusion and heating [3,12], the optical fiber ends in the shape of molten silicon are simultaneously acted by various forces such as applied force, surface tension, thermal expansion force and gravity in the whole fusion-splicing process, as shown in Figure 2b. Although the 
I.Hatakeyama model and the A.K. Das model put forward the force characteristics of the optical fiber ends contacting with each other during the fusion-splicing process on the basis of the force equilibrium theorem, and all have given the optimal fusion conditions to minimize the loss, these models are merely based on plain areas and ignore the gravity effect of optical fiber. When these optimal fusion-splicing conditions are applied to the high altitude environment, they have failed to achieve the expected results, even far exceeded the desired results. So then, we correlate the high altitude environment with these findings and establish a new mathematical model.

In a high-altitude environment, with the increase of altitude $[15,16]$, complex terrain and changeable climate seriously affect the heating and stress state of the optical fiber in the fusion region. When the altitude is $Z$ meters with the $P_{Z}$ pascal intensity of pressure and the $F_{P}$ pressure, the applied force that makes the two optical fibers be squeezed each other and connected together becomes $F_{B}-F_{P}$. According to the thermodynamic theory, the ideal equation of state of gas [15] is $m P=\rho_{a} K T_{0}$, besides, the equation of atmospheric mechanics is $\partial P / \partial Z=-\rho_{a} g$, so the general equation of pressure height is $-\left(m g / K T_{0}\right) \partial Z=\partial P / P$. It should be noted that these are different from the physical mechanism proposed in literature [26]. Furthermore, the equation can be obtained:

$$
P_{Z}=P_{0} \exp \left[\int_{Z_{0}}^{Z}-\left(\frac{m g}{T_{0} K}\right) \partial Z\right]=P_{0} \exp \left[-\left(\frac{m g}{T_{0} K}\right)\left(Z-Z_{0}\right)\right]
$$

where $P_{0}$ is the standard atmospheric pressure at an altitude of $0 ; P$ is the atmospheric pressure; $\rho_{a}$ is the density of air; $m$ is the molecular mass of air; $g$ is the acceleration of gravity; $K$ is the gas constant of dry air; $T_{0}$ is the surface air temperature.

Combining Formula (7), the atmospheric pressure $F_{P}$ on the optical fiber end face with cross-sectional area of $A_{c s a}=\pi(D / 2)^{2}$ in the high altitude environment is:

$$
F_{P}=P_{Z} A_{c s a}
$$

The gravity exerted on the fused fiber is difficult to be ignored in the high altitude environment. Combined with Newton's law of gravitation, the gravity $F_{g}$ on the fiber in the fiber fusion region after derivation can be calculated by the following formula.

$$
F_{g}=\frac{\pi \rho G M l_{C} r^{2}}{(R+Z)^{2}}
$$

where $\rho$ is the density of the optical fiber material; $G$ is the gravitational constant; $M$ is the mass of the earth; $R$ is radius of the earth; $l_{C}$ is the length of the fused region.

Furthermore, the axial offset $s$, tilt angle $\theta$ and end face spacing $d$ between the two different fibers are all affected by the high altitude environment to varying degrees. Then, the splice loss between two different fibers is affected to different degrees.

It should be noted that according to Equation (2), the mode field radius $w$ of the optical fiber does not change with the increase of altitude and is only determined by the material characteristics of the optical fiber itself [14]. In order to highlight the research focus of this paper and eliminate unnecessary interference, it is assumed that the inertia of optical fiber material is very ideal. Therefore, when fusion-splicing is carried out at high altitude, the mode field diameter $2 w$ is not affected by the change of altitude.

According to circular axisymmetric characteristics of single-mode fiber [13], when the axial positions of the two fibers are changed under the influence of high altitude environment, the forces in axial direction of fibers do cancel each other out. So on the basis of the coordinate system shown in Figure 2a, it can be obtained through the derivation that the stress state of fibers at this time is as follows:

$$
k_{1} A_{c s a}\left(F_{B}-F_{P}\right)+F_{C}-F_{g}-\frac{\pi D F_{A} x}{l_{B}}=-\frac{\pi \eta D^{2}}{4 l_{B}} \frac{d x}{d t}
$$


where $k_{1}$ is the constant under the action of the applied force $F_{B} ; D$ is optical fiber diameter; $F_{A}$ is the surface tension of optical fiber; $\eta=0.0769 \exp \left(2.90 \times 10^{4} / T\right)$ is the viscosity of molten glass; the thermal expansion force in the fusion region $F_{C}=2 k_{2} A_{c s a} \alpha l_{C} T$ [9]; $l_{B}$ is the width of the melting zone; $t$ is the fusion-splicing time; $T$ is the fusion-splicing temperature; $l_{C}$ is the length of the melting zone; $k_{2}$ is the constant under the action of thermal expansion; $\alpha$ is the coefficient of thermal expansion.

According to the discharge characteristics of the fusion splicer [3] and the stress distribution characteristics of the fusion splices [5,12], the characteristics of surface of the optical fiber end face in the fusion process can be obtained:

$$
2 \pi l_{A} F_{A}=F_{A 0} \times \exp \left[-\exp \left(12.46-\frac{1.54 \times 10^{4}}{T}\right) t^{0.63}\right]
$$

where $F_{A 0}$ is the initial axial stress; $l_{A}$ is the effective fiber length acted on by $F_{A}$.

In combination with (8)-(11), it can be obtained through the derivation that the lateral offset change $s_{Z}$ of the fiber core at this time is as follows:

$$
s_{Z}=\frac{2 k_{1} l_{B} A_{c s a}\left(F_{B}-F_{P}\right) / D+F_{C}-F_{g}}{2 \pi F_{A}}\left[\exp \left(-\frac{4 F_{A}}{D \eta} t\right)-1\right]+s_{0} \exp \left(-\frac{4 F_{A}}{D \eta} t\right)
$$

Similarly, according to circular axisymmetric characteristics of single-mode fiber [13], the stress state in radial direction of optical fiber is not required to be considered when analyzing the stress state in axial direction of optical fiber. In addition, because optical fiber is kept in horizontal direction, the gravity effect can be ignored. So on the basis of the coordinate system shown in Figure 2a, the stress state of optical fiber at this time can be obtained by derivation.

$$
\Delta A_{c s a}\left(F_{B}-F_{P}\right)+F_{C}-2 \pi l_{A} F_{A}=-\pi \eta \frac{D}{2} \frac{d z}{d t}
$$

where $\Delta A_{c s a}$ is the effective area of the fused fiber under the action of $F_{B}$.

Furthermore, the inclination angle $\theta$ between the fiber end faces changes at this time. Combining with (8), (11) and (13), the value of the offset $\theta_{Z}$ can be obtained through derivation, which is distinctly different from the angle value given in reference [26].

$$
\theta_{Z}=\theta_{0} \exp \left[k_{B}\left(F_{B}-F_{P}\right)\right]^{n}
$$

where $\theta_{0}$ is the initial tilt angle; $k_{B}$ and $n$ are constants, both of which depend on the optical fiber end face state.

When the polynomial on the left side of Equation (13) is less than 0, that is, when the two optical fibers are pulled apart and in the state of "separation", the fusion-splicing does not happen. Moreover, the concave, convex or polluted end face of the optical fiber can lead to an initial end face spacing $d_{0}$. However, only when the distance between two optical fibers is less than a certain value, can the fusion-splicing take place [3]. Therefore, when the gap distance $d$ between the two optical fibers is within the effective range, the dominant role in the whole fusion process is the force exerted by the fusion splicer. So the value of optical fiber end face spacing, $d_{Z}$, is not affected by the change of altitude, but mainly depends on the fusion-splicing time [9], namely:

$$
d_{Z}=d_{0}+\frac{2 F_{A}}{\eta} t
$$

\subsection{Splicing Loss}

In combining with (1), the splicing loss $L$ of two different single-mode optical fibers in high altitude environment can be expressed as:

$$
L=-10 \log f(w, s, \theta, d ; Z)
$$


According to Equation (2), the influence of high altitude on the fusion-splicing of different kinds of optical fibers in ideal state has not been involved. By applying the Gaussian approximation method and combining Equations (3) and (16), it can be obtained that the loss $L(w)=L_{w}(Z)$ only caused by MFD mismatch in the environment of high altitude is as follows:

$$
L_{w}(Z)=-20 \log \left(\frac{2 w_{1} w_{2}}{w_{1}^{2}+w_{2}^{2}}\right)
$$

Substituting Equations (4), (7) and (12) into Equation (16) and applying the Gaussian approximation method, it can be obtained through the derivation that the fusion loss caused by the transverse displacement $s$ of the optical fiber core under the influence of high altitude is, $L(s)=L_{s}(Z)$, namely:

$$
\begin{aligned}
& L_{S}(Z)=-20 \log \left(\frac{2 w_{1} w_{2}}{w_{1}^{2}+w_{2}^{2}}\right)+\frac{8.686}{w_{1}^{2}+w_{2}^{2}} \times \\
& \left\{\frac{2 k_{1} l_{B} A_{c s a}\left(F_{B}-F_{P}\right) / D+F_{C}-F_{g}}{2 \pi F_{A}}\left[\exp \left(-\frac{4 F_{A}}{D \eta} t\right)-1\right]+s_{0} \exp \left(-\frac{4 F_{A}}{D \eta} t\right)\right\}^{2}
\end{aligned}
$$

Similarly, substituting Equations (5), (7) and (14) into (16), the splice loss, $L(\theta)=L_{\theta}(Z)$, caused by the inclination angle $\theta$ between the end faces of two fibers affected by high altitude, can be obtained through the derivation. Importantly, the experimental data show that the results obtained by (19) are more consistent with the actual situation in high altitude environment than those obtained by Formula (12) in Reference [26].

$$
L_{\theta}(Z)=-20 \log \left(\frac{2 w_{1} w_{2}}{w_{1}^{2}+w_{2}^{2}}\right)+\frac{8.686 \pi^{2} n_{i}^{2} w_{1}^{2} w_{2}^{2}}{\lambda^{2}\left(w_{1}^{2}+w_{2}^{2}\right)} \sin ^{2}\left\{\theta_{0} \exp \left[k_{B}\left(F_{B}-F_{P}\right)\right]^{n}\right\}
$$

When the effect of applied force and atmospheric pressure and the thermal expansion of molten silicon are weaker than that of the surface tension of optical fiber end face, the Fresnel reflection loss accounts for a small part [18]. However, in view of the research focus of this paper, the Fresnel reflection loss is ignored here. According to Equations (6), (15) and (16) and applying the Gaussian approximation method, it can be obtained that the fusion loss, $L(d)=L_{d}(Z)$, caused only by end face spacing $d$ under high altitude environment, is as follows:

$$
L_{d}(Z)=-10 \log \frac{4 w_{1}^{2} w_{2}^{2}}{\left(w_{1}^{2}+w_{2}^{2}\right)^{2}+\left[\lambda\left(d_{0}+2 F_{A} t / \eta\right) / \pi n_{i}\right]^{2}}
$$

The $L_{w}(Z), L_{s}(Z), L_{\theta}(Z)$, and $L_{d}(Z)$ respectively represent the splicing loss of two different kinds of optical fibers under the condition of MFD mismatch, axial offset, angle tilt or end face gap in the high altitude environment. According to the independence of these four influence factors, the total splice loss $L$ can be obtained.

$$
L=L(\text { loss })=L_{w}(Z)+L_{s}(Z)+L_{\theta}(Z)+L_{d}(Z)
$$

In the high altitude environment with harsh and changeable climate as well as a complex train, it is difficult for human to stay for a long time. The actual measurement proves that the instruments and apparatuses are easy to fail in this environment. Besides, the electrodes of the fusion splicer are easily polluted. What gives rise to these problem is not the focus of this paper, and therefore can not be described here. However, these factors can lead to some deviation between actual fusion loss and theoretical values, which is described here as additional loss and is expressed by the symbol $L_{a d}$. Obviously, the additional loss $L_{a d}$ can increase or decrease the total value of splicing loss [3]. In order to 
make more accurate calculation and comparison, the splicing loss obtained under high altitude environment is further expressed as:

$$
L_{\text {tot }}=L(\text { loss })+L_{a d}
$$

\section{Experiments}

\subsection{The High and Cold Plateau Region}

So far, the field experiments relating to fiber fusion under harsh weather conditions are too few and incomplete. To worsen the situation, the experimental data concerning fiber splice which can be utilized for analysis and application under high altitude environment are seriously insufficient. In order to facilitate the understanding and calculation, this paper draws on engineering experience and defines the day with $-25^{\circ} \mathrm{C}$ in one day as the high cold day, and the month of 5 high cold days in one month as the high cold month, and the climate of 3 high cold months in a year as the high cold region. Besides, the area with an altitude of $3000 \mathrm{~m}+$ is defined as a plateau. Evidently, the high and cold plateau region is a typical high altitude region with extremely harsh environment. Since accurate control of fusion parameters is essential for successful splicing [3,22], the fusion process is easily affected by the environment. In order to achieve a satisfactory splice result, multiple parameters often need to be carefully controlled simultaneously. Up to present, Hatakeyama, Das and Kato et al., combining with different fusion conditions in plain area, have found the corresponding optimal parameter combinations to minimize splice loss. For example, One of the optimal fusion conditions is the $2000^{\circ} \mathrm{C}$ fusion temperature, $18 \mathrm{~mA}$ discharge current, $8.5 \mathrm{~W}$ discharge power, $1 \mathrm{~s}$ heating time, and $20 \mathrm{~g} \cdot \mathrm{m}$ applied force. Consequently, the optimal splice theory and experience $[6-11,21,23]$ are initially utilized to conduct field experiments in the high and cold plateau region.

Two kinds of standard single-mode-fiber (SMF), referring to G.652.D and ultra-lowloss (ULL) [27] fiber, are selected as research objects. It should be noted that these two optical fibers in the subsequent fusion experiment are from the same manufacturer, same batch and same model respectively. In order to eliminate unnecessary interference and facilitate calculation, the average values of material parameters and that of geometric parameters of these two optical fibers are taken respectively, and the detailed parameters are shown in Table 1. Besides, they all are "step type" single-mode fibers. According to splice theory, the "ULL-ULL" mode and "G652D-G652D" mode belong to the category of the same kind of fiber fusion, while "ULL-G652D" mode is opposite.

Table 1. Optical fiber characteristic parameters.

\begin{tabular}{ccccc}
\hline Fiber Type & $\mathbf{r}(\boldsymbol{\mu m})$ & $\mathbf{D}(\boldsymbol{\mu m})$ & $\mathbf{A}\left(\boldsymbol{\mu \mathbf { m } ^ { 2 } )}\right.$ & MFD $(\boldsymbol{\mu m})$ \\
\hline G.652.D & 4.2624 & 125.3734 & $12,345.31$ & 10.3624 \\
ULL & 4.3125 & 125.1216 & $12,296.47$ & 10.257 \\
\hline
\end{tabular}

In order to exclude the interference of the optical fiber itself, 10 samples at a time are taken from each selected experimental object during the experiment, and it is assumed that the samples and its parameters are consistent in the same sample space. Moreover, in order to compare and analyze the experimental results more accurately, the subsequent fusion experiments are repeated in accordance with the same number of experiments and the same steps.

\subsection{Areas of Different Altitudes}

Based on China's "Tibet-middle part" networking project, fusion experiments are carried out at seven different altitudes. Fortunately, the altitude have already been accurately measured $[15,16]$. The environmental factors at different altitudes are different, and the environmental information of these seven altitudes is shown in Table 2. It should be noted that a large number of engineering experience shows that lightning, snowfall and rainfall 
are not the main factors affecting the splice loss. Therefore, we only consider the meteorological factors such as temperature, humidity, oxygen content, atmospheric pressure, gale and gravity. During the whole experiment, the length of each optical fiber of G652D and ULL all is $500 \mathrm{~m}$. Except for the special adjustment of the follow-up experiments, all the fusion-splicing experiments in this paper select SM G652 Std. fusion-splicing mode and choose the default parameters of the fusion splicer manual as the parameters in the fusion-splicing process. After the fusion-splicing is completed successfully, the Optical Time Domain Reflectometer (OTDR) is utilized to measure the splice loss at the wavelength of $1550 \mathrm{~nm}$. The EXFO MAX-730B-M2 OTDR, which is widely used in business at present, is selected in this paper. OTDR is similar to a radar when reading experimental data. It first sends out a test laser pulse to the optical fiber and then observes the size of the laser power returning from each point on the optical fiber (including Rayleigh scattering and Fresnel reflection). This process is repeated. Then, these results are averaged according to the needs and displayed in the form of trajectory diagram, which describes the instantaneous situation of the whole section of the optical fiber. Given the OTDR "left-right" and "right-left" two features of the measuring way of the proceeds of the results are not the same [28], and also in order to eliminate the unnecessary disturbance and be convenient for numerical calculation, this paper in all of the fusion-splicing experiments adopts "two-way measurement" way of measuring the results of each experiment to measure and then takes the average as the final loss values.

Table 2. The environmental information at different altitudes.

\begin{tabular}{cccccccc}
\hline Altitude $(\mathbf{m})$ & $\mathbf{5 3}$ & $\mathbf{2 9 8 0}$ & $\mathbf{4 0 0 0}$ & $\mathbf{4 2 0 0}$ & $\mathbf{4 3 0 0}$ & $\mathbf{5 0 2 0}$ & $\mathbf{5 2 0 0}$ \\
\hline temperature $\left({ }^{\circ} \mathrm{C}\right)$ & 24 & 24 & 13 & 12 & 13 & 20 & 18 \\
humidity $(\% \mathrm{RH})$ & 54 & 50 & 66 & 60 & 66 & 50 & 50 \\
oxygen content $(\%)$ & 20 & 15 & 12 & 14 & 13 & 10 & 9 \\
wind speed $(\mathrm{m} / \mathrm{s})$ & 2 & 14 & 24 & 29 & 27 & 19 & 17 \\
\hline
\end{tabular}

In order to eliminate the interference caused by optical fiber cutting machine, optical fiber wiping paper, optical fiber fusion splicer, OTDR measuring instrument or other equipment, this paper assumes that all the tools, instruments and meters utilized in the fusion-splicing process are clean and in good condition, besides, they all are also very ideal in the process of application.

\subsection{Different Locations in the Same Altitude Environment}

In experiment II, the fusion losses at different altitudes have been compared in detail. However, the environmental factors at different locations at the same altitude are different [15]. So then, we select the most typical two altitudes for further experiments, and the environmental information of different locations at the same altitude is shown in Table 3. Obviously, the area with an altitude of $53 \mathrm{~m}$ is a typical plain area, while the area with an altitude of $4300 \mathrm{~m}$ is a typical high altitude area. The fusion-splicing experiments are respectively carried out in four different places with an altitude of $53 \mathrm{~m}$ and in four different places with an altitude of $4300 \mathrm{~m}$ under the same conditions.

Table 3. Environmental information of different locations at the same altitude.

\begin{tabular}{ccccccccc}
\hline \multirow{2}{*}{ Environmental Information } & \multicolumn{3}{c}{ 53 m above Sea Level (Plain Area) } & \multicolumn{3}{c}{ Altitude 4300 m (High Altitude Area) } \\
\cline { 2 - 8 } & Location $\mathbf{1}$ & Location 2 & Location 3 & Location 4 & Location 1 & Location 2 & Location 3 & Location 4 \\
\hline temperature $\left({ }^{\circ} \mathrm{C}\right)$ & 18 & 21 & 24 & 27 & 13 & 15 & 13 \\
humidity $(\% \mathrm{RH})$ & 52 & 41 & 54 & 56 & 65 & 67 & 66 & 61 \\
oxygen content $(\%)$ & 20.5 & 19.5 & 20 & 20.5 & 12.5 & 13.5 & 13 & 12 \\
wind speed $(\mathrm{m} / \mathrm{s})$ & 1.7 & 1.7 & 2 & 2.1 & 25.6 & 26.7 & 27 & 28 \\
\hline
\end{tabular}


It is worth noting that, although the MFD of optical fiber is extremely important and thus its mismatch can cause a large fusion loss [17,19], the theory discussed above and the later simulation results all show that the high altitude has no effect on MFD. After excluding the influence of the MFD mismatch on the splice loss in the high altitude environment, we only need to select the same kind of optical fiber for further experiments to achieve the purpose, that is, what is the influence of high altitude environment on the splice loss in the following research. In consideration of the demand of the "Tibet-middle part" networking project, the ULL fiber is only selected as the further experimental object in the subsequent research.

\section{Results and Discussion}

Figure $2 \mathrm{~b}$ shows that two optical fibers are squeezed together during fusion-splicing due to the joint action of various forces, including $F_{A}, F_{B}, F_{C}, F_{P}, F_{g}$ and glass viscosity. Meanwhile, Figure 3 shows that the splice loss is mainly caused by MFD mismatch, axial offset, angle tilt or end face gap in the fusion process. According to the second part of this paper, the corresponding splice loss varies with the changing force being applied on the two fibers, as shown in Figure 4. The unit of the resultant force is $\mathrm{g} \cdot \mathrm{m}$. Obviously, the fusion process is controlled by the fusion splicer. Under ideal conditions, the effective range of applying force for achieving the lowest splice loss is given by I.Hatakeyama as 20 30 g.m, while A.K. Das also indicates that the effective range of applying force to achieve the lowest splice loss is $20 \sim 25 \mathrm{~g} \cdot \mathrm{m}$. So in the environment of high altitude, the range of the applied force is set from the lowest value to the critical value of $30 \mathrm{~g} \cdot \mathrm{m}$ under ideal conditions. As can be seen from Figure 4, the green curve with "*” and the water red curve with " $\circ$ " all have no significant change, which indicates that the fusion loss $L(w)$ or $L(d)$ caused by the MFD mismatch or the end face spacing $\mathrm{d}$ does not change with the change of applied force, while the blue curve with " $\triangle$ " and the red curve with " $\square$ " all emerge as an obvious upward trend, which indicates that the fusion loss $L(s)$ or $L(\theta)$ caused by the axial deviation $s$ or inclination angle $\theta$ increases evidently with the increase of the applied force. Besides, $L(s)$ is larger than $L(w), L(d)$ and $L(\theta)$, while $L(\theta)$ increases significantly with the increase of applied force under ideal conditions.

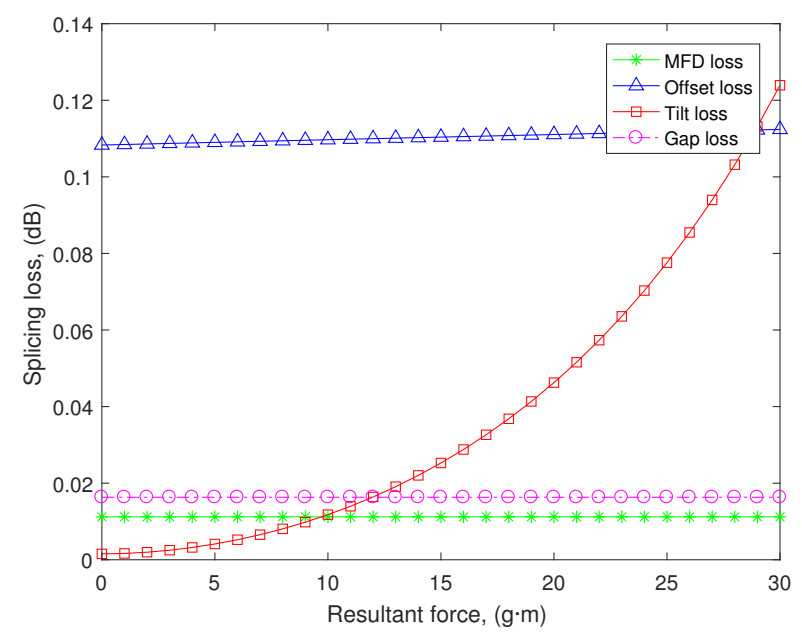

Figure 4. Splice loss versus resultant force, the four fusion variables affected by the resultant force have an impact on the splice loss of two kinds of optical fibers. In high altitude environment, the resultant force includes surface tension, the force exerted by the fusion splicer, atmospheric pressure, gravity force, the thermal expansion force and glass viscosity force, and increases in steps size of $1 \mathrm{~g} \cdot \mathrm{m}$, as shown in the abscissa of the picture.

According to Equations (3)-(6), the main factors that cause fusion loss are MFD mismatch, axial offset, angular tilt or end face spacing. Meanwhile, it can be seen from Equations (2), (7), (12), (14) and (15) that, in high altitude environment, the mode field 
diameter $2 w$, axial offset $s$, inclination angle $\theta$ or end face spacing $d$ of the two optical fibers can be plotted to vary with the increase of altitude, as shown in Figure 5. Since the units of $w, s, \theta$ and $d$ are not exactly the same, and the amount of change is also inconsistent, the scale of the corresponding ordinate is here set to be not consistent, as shown in Figure 5. It is assumed that the properties of optical fiber materials are relatively stable in the environment of high altitude, and according to Equation (2), the mode field radius of optical fiber does not change with altitude. Therefore, the $w_{g}$ of G.652.D and $w_{u}$ of ULL fibers all do not change with increasing altitude, and $w_{g}>w_{u}$, as shown in Figure 5a. According to Equations (12) and (14), the values of axial offset and tilt angle all vary with altitude. For the convenience of comparison and drawing, the variation is used to represent the changes of axial offset and angular tilt with altitude. Then, the axial offset change $\Delta s$ and tilt angle change $\Delta \theta$ between two optical fibers all show an obvious upward trend with the increase of altitude, as shown in Figure $5 b$,c. It is worth noting that the axial offset change $\Delta s$ must be less than $\min \left\{r_{L}, r_{R}\right\}$ and the tilt angle change $\Delta \theta$ is less than 90 degrees. Otherwise, it is difficult to splice successfully, or the loss of joint splice successfully is also very huge to be unacceptable. Besides, the Equation (15) shows that, although the end face spacing $d$ between two optical fibers can change during the fusion-splicing process, it does not change with the increase of altitude. Therefore, the change trend of end face gap with altitude is shown in Figure 5d. Furthermore, comparing (a), (b), (c) and (d) in Figure 5 and combining with the Formulas (17)-(20), it can be concluded that Mold field diameter mismatch, axial offset, angle tilt and end face gap can all affect the splice loss, but the axial offset and angular tilt are greatly affected by the high altitude and then have the most tremendous influence on the splice loss between two kinds of optical fibers at high altitudes. In order to highlight the core of this study, it only needs to next focus on the analysis of the influence of the axial offset or angle tilt between two optical fibers affected by the high altitude environment on the fusion loss.
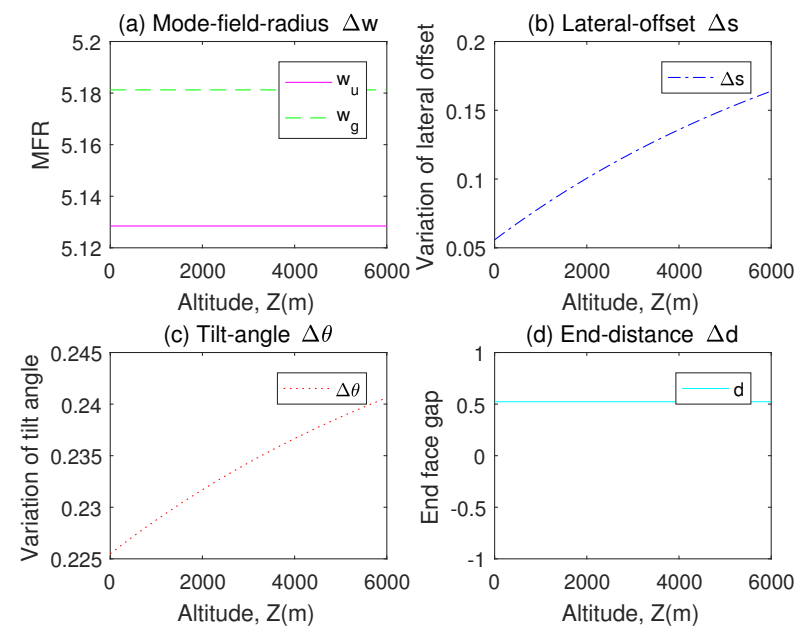

Figure 5. Fusion variables versus altitudes, the abscissa represents the altitude and increases in steps size of $10 \mathrm{~m}$, and the ordinate represents four fusion variables affected by the high altitude environment. (a): the mode field radii of G.652.D and ULL fibers are $w_{g}$ and $w_{u}(\mu \mathrm{m})$ respectively, and variation $\Delta w$ is 0 ; (b):variation of lateral offset $\Delta s(\mu \mathrm{m}) ;(\mathbf{c})$ :variation of tilt angle $\Delta \theta$ (degrees); (d): the end face spacing of two optical fibers, and variation $\Delta d(\mu \mathrm{m})$ is 0 .

It has been shown that the axial offset or angular tilt is more affected by the high altitude environment and also has a greater impact on the fusion loss than the MFD mismatch or end face spacing between fibers in that environment. So in order to more accurately verify the correctness of the proposed method, in the high altitude environment, this paper next carries out multiple groups of repeated experiments for three fusion methods consisting of "ULL-ULL", "G652D-G652D" and "ULL-G652D" under the same conditions. The experimental results show that, in the case of MFD mismatch, although the value of UG is 
greater than that of UU and GG, the three curves of UU, GG and UG all do not change, as shown in Figure 6a, which indicates that the fusion loss $L(w)$ caused by MFD mismatch is not affected by the high altitude environment. However, when only lateral offset occurs, although the value of UG is greater than that of UU and GG, only attributing to MFD mismatch, the three curves of UU, GG and UG all show a consistent upward trend, without obvious difference, as shown in Figure $6 \mathrm{~b}$. This result shows that the fusion loss $L(s)$ only caused by axial offset increases when being affected by the high altitude environment, but does change greatly not because of MFD mismatch. When only angle tilt occurs, although the value of UG is greater than that of UU and GG, only attributing to MFD mismatch, the three curves of UU, GG and UG all show a consistent upward trend, with no obvious difference, as shown in Figure 6c, which shows that the fusion loss $L(\theta)$ caused only by the tilt angle increases when being affected by the high altitude environment, but does change greatly not because of MFD mismatch. Similarly, when only end face gap occurs, although the value of UG is greater than that of UU and GG, the three curves of UU, GG and UG all do not change, as shown in Figure 6d, which indicates that the fusion loss $L(d)$ only caused by end face spacing is not affected by the high altitude environment and also does change greatly not due to MFD mismatch. It's very important that these results are basically consistent with the results given by Formulas (18) and (19). Therefore, it can be verified again that the splice loss caused by MFD mismatch or end face gap is not affected by the high altitude environment under any conditions. In addition, by comparing (b) and (c) in Figure 6, it is found that the curve of $L(s)$ increases sharply at first and then slowly, while the curve of $L(\theta)$ almost always increases steadily. This difference indicates that $L(s)$ and $L(\theta)$ are all affected by altitude, but to the different extent, under different conditions.
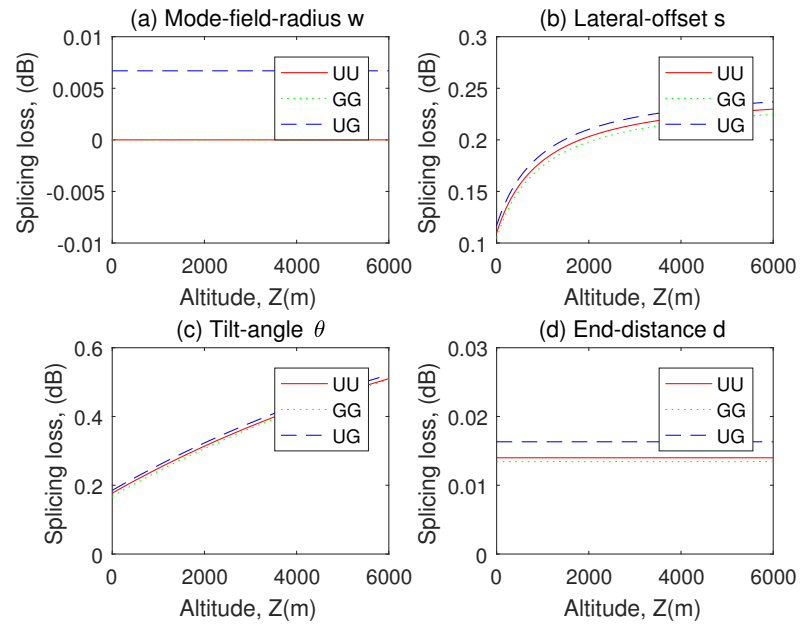

Figure 6. Splice loss versus altitudes, under the same ideal conditions, the four fusion variables affected by the high altitude environment have an impact on the splice loss of two kinds of optical fibers, and the abscissa represents the altitude and the ordinate represents the splice loss. The length of G652D fiber and ULL fiber are both $500 \mathrm{~m}$, and their characteristic parameters are described in Table 1. "UU" means "ULL-ULL"; "GG" means "G652D-G652D"; and "UG" means "ULL-G652D". Under these three different fusion modes, (a):MFD mismatch; (b):lateral offset; (c):angle tilt; (d):end face gap, as shown in Figure 3.

In order to verify the accuracy of the model proposed, the "ULL-ULL" mode is selected for further analysis. In the coordinate system we established, the altitude increases in steps size of $10 \mathrm{~m}$ as the altitude increases continuously, as shown in Figure 7. Combining with Equations (17)-(20), in an ideal condition, the fusion loss caused by axial deviation or angular inclination between the two optical fibers increases significantly, while the fusion loss caused by MFD mismatch or end face spacing does not change, which indicates that $L(s)$ and $L(\theta)$ are susceptible to the high altitude environment, while $L(w)$ and $L(d)$ are not affected by the same environment. In addition, the curve of $L(s)$ increases sharply at 
first, then slowly, and finally even levels off, while the curve of $L(\theta)$ almost always tends to increase steadily, which indicates again that $L(s)$ and $L(\theta)$ all are affected by altitude, but to the different extent.

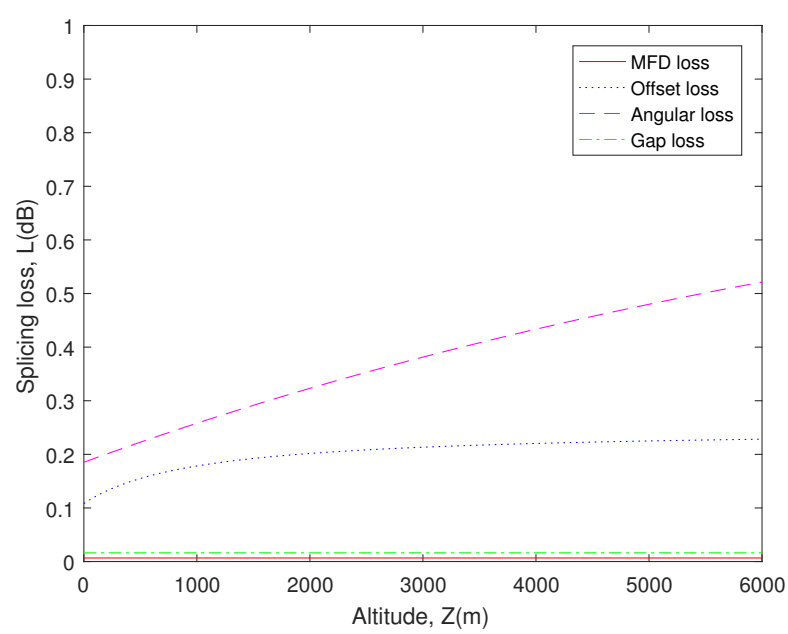

Figure 7. Splice loss versus altitudes, the four fusion variables affected by the high altitude environment have an impact on the splice loss of ULL fiber.

By comparing Figures 5-7, it is found that the MFD mismatch, axial offset, angle tilt and end face gap as shown in Figure 3 all cause the fusion loss between optical fibers to a large extent. However, under ideal conditions, according to Equations (2) and (15), $w$ and $d_{Z}$ are not affected by the high altitude environment, while according to Equations (12) and (14), $s_{Z}$ and $\theta_{Z}$ are greatly affected by the high altitude environment. Therefore, in order to cut down the splice loss at high altitudes, we should focus on how to reduce the $s_{Z}$ and $\theta_{Z}$ in the future research.

In order to further verify the applicability and accuracy of the model proposed, we further compare it with I.Hatakeyama model, D. Marcuse model and A.K. Das model, as well as with the initial and adjusted experimental results respectively, as shown in Figure 8. Obviously, it can be seen from the simulation diagram that, D.Marcuse curve, I.Hatakeyama curve and A.K. Das curve all tend to be stable under the high altitude environment. Meanwhile, The average splice loss of $0.62 \mathrm{~dB}$ obtained by the D. Marcuse model is the largest, while that of $0.56 \mathrm{~dB}$ coming from the I.Hatakeyama model is reduced by $0.09 \mathrm{~dB}$, besides, that of $0.53 \mathrm{~dB}$ obtained by the A.K. Das model is reduced to about $90 \%$ of the original loss. However, the fusion losses obtained by these models in the high altitude environment under the same conditions all are stable at a very high level continually and greatly exceed the loss budget, which is very difficult to accept. The red curve in the figure shows that the fusion loss increases with the change of altitude $Z$, but it is significantly smaller than the results obtained by these above models, which further verifies the applicability of the model proposed whose average is $0.42 \mathrm{~dB}$. In addition, the theoretical values obtained by utilizing the model presented in this paper are obviously smaller than the initial experimental results with the average value of $0.51 \mathrm{~dB}$ indicated by the symbol " $\circ$ ", but are evidently close to the experimental results after processing with that of $0.43 \mathrm{~dB}$ indicated by the symbol " $\triangle$ ", and importantly, the splice loss is reduced by at least $0.07 \mathrm{~dB}$ at this time, which again verifies the correctness of the model presented. However, when the altitude is higher than $5200 \mathrm{~m}$, the measured losses are obviously greater than the theoretical values. To make matters worse, this phenomenon becomes more and more obvious with the increase of altitude. It needs to be noted that, the deviation of the experimental results, can be due to the failure of instruments and apparatuses such as the light source, optical power meter, fusion splicer or OTDR when they are working in the high altitude environment and exceeding the applicable range specified in their specifications (the effective working range is less than $5000 \mathrm{~m}$ generally). It is particularly 
obvious that the points with large deviation from the theoretical values occur especially when the altitude is higher than $5200 \mathrm{~m}$.

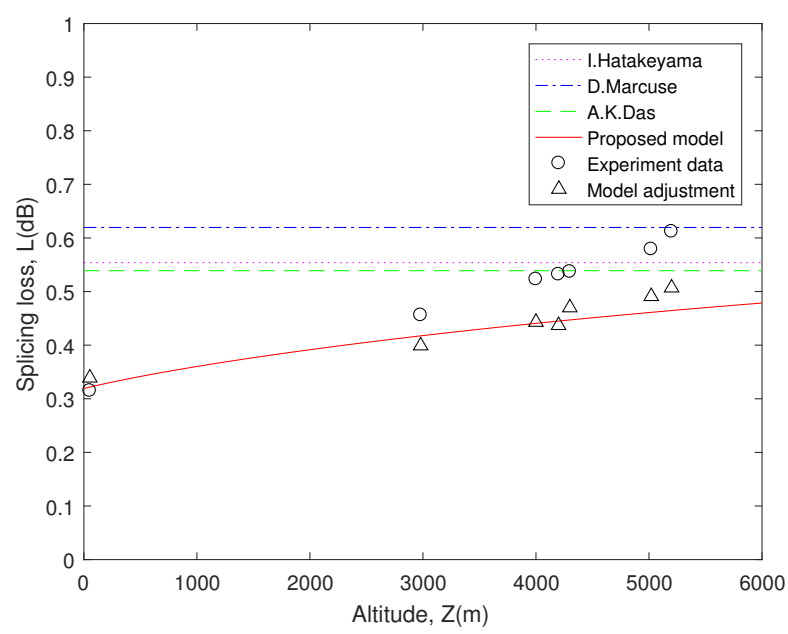

Figure 8. Splice loss versus altitudes, the theoretical values are obtained according to the optimum fusion conditions given by the respective models, and the field fusion-splicing has been carried out in the high altitude environment.

In order to further verify the practicability of the model proposed in the high altitude environment under the same settings, the experiments have been carried out in the areas with altitudes of 53,2980,4000, 4200, 4300, 5020 and $5200 \mathrm{~m}$ respectively, as described in Experiment II. The measured results of the splice loss obtained are shown in Figure 9. Obviously, the average splice loss of $0.08 \mathrm{~dB}$ at the altitude of $2980 \mathrm{~m}$ is greater than that of $0.037 \mathrm{~dB}$ at the altitude of $53 \mathrm{~m}$ (plain), while that of $0.27,0.30$, and $0.33 \mathrm{~dB}$ at the altitude of 4000, 4200, and $4300 \mathrm{~m}$ all are greater than that of $0.08 \mathrm{~dB}$ at the altitude of $2980 \mathrm{~m}$. In addition, the splice losses at the altitude of 4000, 4200, and $4300 \mathrm{~m}$ tend to increase with altitude. Moreover, the average splice loss of 0.44 and $0.47 \mathrm{~dB}$ at the altitude of 5020 and $5200 \mathrm{~m}$ are greater than that of $0.27,0.30$, and $0.33 \mathrm{~dB}$ at the altitude of 4000 , 4200 , and $4300 \mathrm{~m}$. Therefore, it can be concluded that the splice loss between two fibers can also increase dramatically with the increase of altitude in the high altitude environment. Besides, the average fusion loss of $0.08 \mathrm{~dB}$ at an altitude of less than $3000 \mathrm{~m}$ is significantly lower than that of $0.26 \mathrm{~dB}$ at an altitude of more than $4000 \mathrm{~m}$. When the altitude is higher than $5000 \mathrm{~m}$, the average fusion loss increases significantly. The average fusion loss of $0.43 \mathrm{~dB}$ at this time is especially much larger than that of $0.31 \mathrm{~dB}$ at an altitude of less than $4300 \mathrm{~m}$. Evidently, the splice loss increases with the ascending of altitude. These results are basically consistent with those obtained by Equation (21). Consequently, this once again verifies the practicability of the model proposed in the high altitude environment.

In order to further verify the accuracy of the model proposed, further comparisons are made in four different locations at an altitude of $53 \mathrm{~m}$ (plain) and four different locations at an altitude of $4300 \mathrm{~m}$ respectively, as shown in Figure 10. The environmental information at the elevation of 53 and $4300 \mathrm{~m}$ has been given in Table 3. Obviously, the splice losses in four different locations at an altitude of $4300 \mathrm{~m}$ all are higher than that in four different locations at an altitude of $53 \mathrm{~m}$. Besides, the splice losses in four different locations at an altitude of $53 \mathrm{~m}$ are very close with slight fluctuations, while the splice losses in four different locations at an altitude of $4300 \mathrm{~m}$ are also very close, but the splice losses in these four different locations fluctuate greatly. This is largely attributed to the fact that the environmental parameters of different locations at the same altitude are different. This feature is particularly prominent at high altitudes. Importantly, these results are basically consistent with those obtained by the model constructed. 


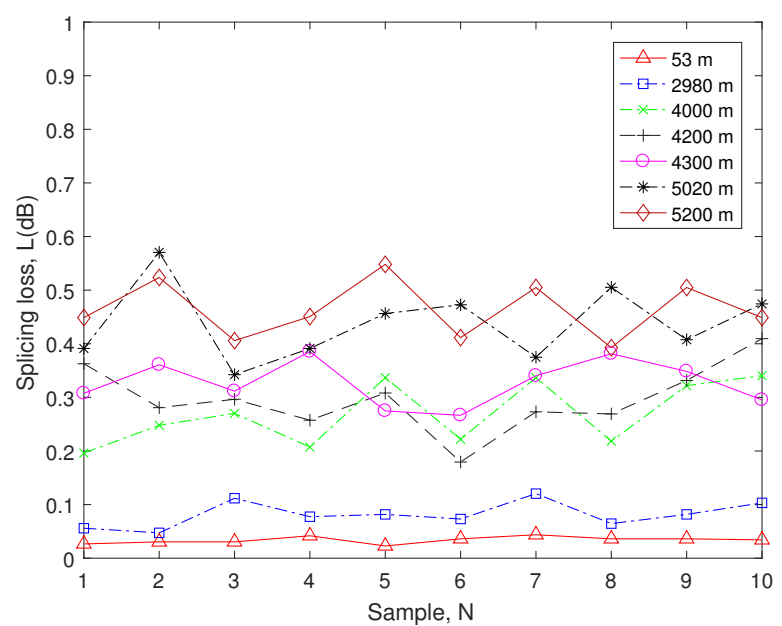

Figure 9. Splice loss versus samples, In the high altitude environment at different altitudes, under the same conditions, the ULL optical fibers have been fused on site, and 10 samples have been taken from each experimental point, and then the average value of the results has been taken.

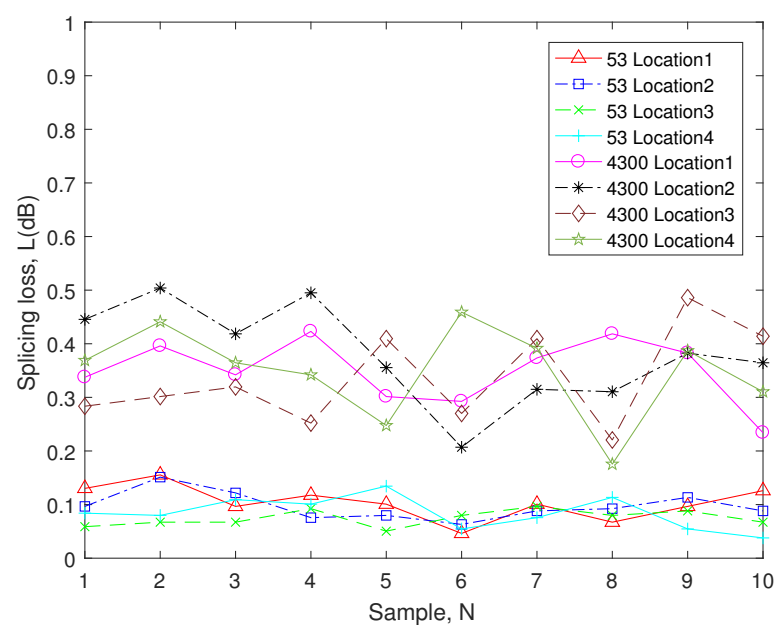

Figure 10. Splice loss versus samples, in four different locations with an altitude of $53 \mathrm{~m}$ (plain) and in four different locations with an altitude of $4300 \mathrm{~m}$ respectively, under the same conditions, the ULL optical fibers have been fused on site, and 10 samples have been taken from each experimental point, and then the average value of the results has been taken.

By comparing Figures 8-10, It can be confirmed that the splice loss between the two optical fibers increases with the rise of altitude in high altitude environment, and the higher the altitude, the greater the splice loss. Besides, the results respectively obtained by the optimal conditions of D.Marcuse model, I.Hatakeyama model and A.K. Das model all are obviously greater than $0.5 \mathrm{~dB}$ or even greater. However, the results obtained by the model constructed in this paper are significantly less than $0.5 \mathrm{~dB}$, and the reduction is at least $0.07 \mathrm{~dB}$. More importantly, when it is applied to the splicing engineering in high altitude environment, the results coming from OTDR are basically consistent with the theoretical values.

The second part of this article shows that, although the main reasons for the splice loss between the two optical fibers are MFD mismatch, axial offset, angle tilt or end face gap, the axial offset and angle tilt are greatly affected by the high altitude under the process of fusion-splicing and hence have a greater impact on the splicing loss in the high altitude environment. Moreover, the experiments mentioned above already prove that the axial offset $s$ and inclination angle $\theta$ are greatly affected by the high altitude and have a greater 
influence on the splice loss. Therefore, in order to further verify the model proposed in this paper, the MFD $2 w$ and end face spacing $d$ all are fixed next, with only the value $s$ or $\theta$ changed.

Figure 11 shows that, at $\theta=1^{\circ}$, when the axial offset is $1 \mu \mathrm{m}$, the measured values at high altitude approach to the theoretical curve. When the alignment between the two optical fibers is ideal (i.e., there is no axial offset), the measured loss instead is far away from the theoretical value. A big reason for this phenomenon is that, in order to achieve good alignment, the splice joint appears "deformation" or "bulge", which then leads to the increase of loss, for instance, bringing in microbending loss and so on at this moment. The theoretical value, nevertheless, is reduced, which once again verifies the correctness of the theoretical model. However, when the axial offset is $4 \mu \mathrm{m}$, the degree of change of the theoretical value instead becomes smaller, because the effective area of fiber exposed in the fusion process is also significantly increased. At this time, the reaction force the two optical fibers experience when they are squeezed together is also increased, but the splice points are prone to produce "bubbles", which then leads to large air gap loss.

Figure 12 shows that, at $\theta=2^{\circ}$, the measured values at high altitude all deviate from and are even greater than the theoretical value, regardless of the axial deviation $\mathrm{s}=1 \mu \mathrm{m}$, $\mathrm{s}=4 \mu \mathrm{m}$, or the ideal axial alignment. This proves once again that, the splice loss caused by the end face tilt is greatly affected by the high altitude. Because of the increase of the tilt angle of the optical fiber end face and the special environment of the high altitude at this time, on the one hand, the "Fresnel reflection" loss between the two optical fibers can not be ignored, and on the other hand, the filling material between the two optical fibers is no longer defaulted as the air with refractive index of 1 . What's more, it is very difficult to accurately control the cutting angle of the end face of the optical fiber [21,29], especially to make $\theta<1^{\circ}$, and it is even more difficult to achieve this precision in the high altitude environment.
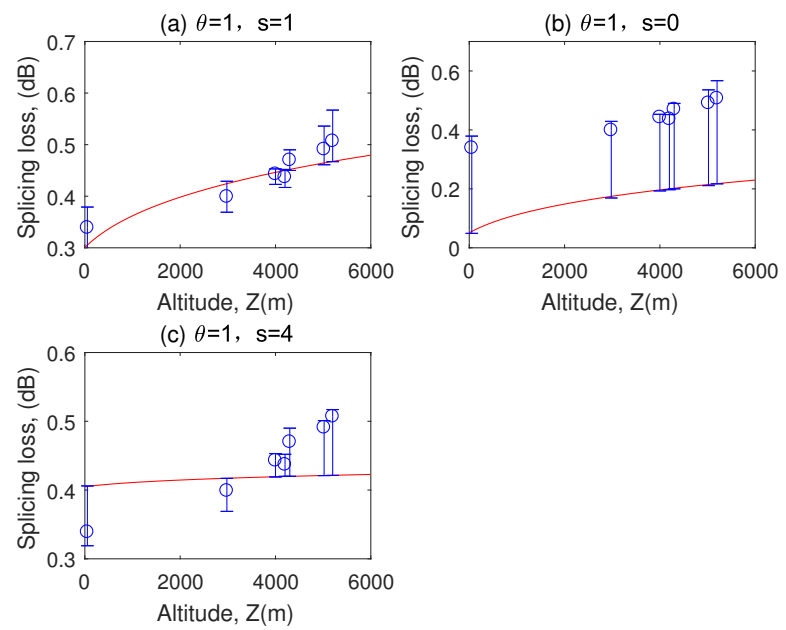

Figure 11. Splice loss versus angular tilt and lateral offset, when the inclination angle $\theta$ is $1^{\circ}$, the axial offset $s$ is different, the red solid line represents the results obtained from the proposed model, and the vertical line with "o" represents the experimental data in the environment of high altitude. The fusion experiments have been carried out at seven locations with different elevations respectively: 53 (plain), 2980, 4000, 4200, 4300, 5020, and $5200 \mathrm{~m}$. After slight modification of the model, (a) $s=1 \mu \mathrm{m}$; (b) $s=0 \mu \mathrm{m}$ and (c) $s=4 \mu \mathrm{m}$ when $\theta=1^{\circ}$.

In addition, comparing Figure 11 with Figure 12, it is found that, at $s=1 \mu \mathrm{m}$, when the tilt angle $\theta$ between the two optical fibers is $1^{\circ}$ or $2^{\circ}$, the measured results are obviously deviated from the theoretical values, while when $\theta=2^{\circ}$, the loss results deviate to a greater extent. Moreover, this phenomenon becomes more obvious with the increase of altitude. At $s=0 \mu \mathrm{m}$, when the tilt angle $\theta$ is $1^{\circ}$ or $2^{\circ}$, the measured results are also obviously deviated from the theoretical values, but when $\theta=1^{\circ}$, the loss results deviate to a greater extent yet, 
which indicates that when there is no lateral offset, the inclination angle between the end faces of two optical fibers is greatly affected by the high altitude environment and thus has a great influence on the splice loss. At $s=4 \mu \mathrm{m}$, when the tilt angle $\theta$ is $1^{\circ}$ or $2^{\circ}$, the loss results obtained in the plain area are all over $0.4 \mathrm{~dB}$, and the splice loss between the two optical fibers increases significantly with the increase of altitude, which easily leads to failure of fusion-splicing at this time. It is also verified once again that the splice loss caused by the axial offset is greatly affected by the high altitude. In particular, the lateral offset $s$ and angle tilt $\theta$ all have a great influence on the splice loss in the high altitude environment. Furthermore, the influence of transverse offset $s$ between the two optical fibers on the splice loss is even greater than the influence of the angle tilt $\theta$ between the two optical fibers on splice loss under the same conditions, because the lateral offset and angular inclination are affected by the high altitude environment to different degrees.
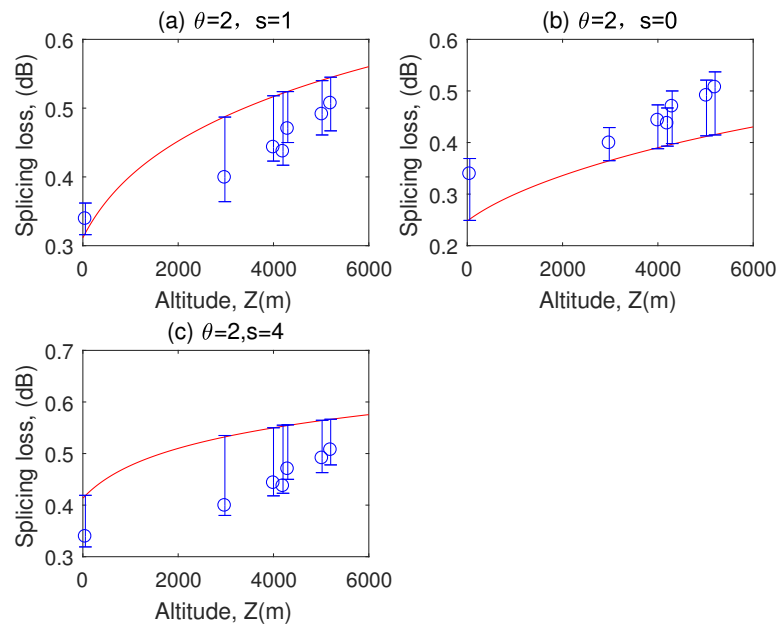

Figure 12. Splice loss versus angular tilt and lateral offset, when the inclination angle $\theta$ is $2^{\circ}$, the axial offset $s$ is different, the red solid line represents the results obtained from the proposed model, and the vertical line with " $\circ$ " represents the experimental data in the environment of high altitude. The fusion experiments have been carried out at seven locations with different elevations respectively: 53 (plain), 2980, 4000, 4200, 4300, 5020, and $5200 \mathrm{~m}$. After slight modification of the model, (a) $s=1 \mu \mathrm{m}$; (b) $s=0 \mu \mathrm{m}$ and (c) $s=4 \mu \mathrm{m}$ when $\theta=2^{\circ}$.

\section{Conclusions}

This paper analyzes the main cause of the splice loss between two different singlemode fibers and discusses the influence of fusion-splicing parameters on splice loss. It is the first time that, the exploratory experiment of field fusion-splicing is carried out in high altitude area. The problem is either it is difficult to splice successfully, or most of the measured loss values are more than $0.5 \mathrm{~dB}$, which greatly exceeds the loss budget. To tackle this problem, we propose a mathematical model to reduce the loss of single-mode fiber fusion under high altitude environment by combining factors of temperature, humidity, oxygen content, atmospheric pressure, strong wind and gravity. Moreover, the main causes of high splice loss in high altitude environment are analyzed and identified. Furthermore, based on China's "Tibet-middle part" networking project, the fusion experiments are for the first time carried out at seven locations with different elevations respectively: 53, 2980, 4000, $4200,4300,5020$, and $5200 \mathrm{~m}$. In addition, fusion experiments still are respectively carried out and compared in four different locations with an altitude of $53 \mathrm{~m}$ and four different locations with an altitude of $4300 \mathrm{~m}$. In particular, a large number of field experimental data have been obtained. What is particularly important is that most of the measured values of splice loss are less than $0.5 \mathrm{~dB}$, and even the loss of some individual splice joints are reduced to about $0.3 \mathrm{~dB}$. The simulation results are basically consistent with the theoretical analysis.

We also found that, in order to reduce splice losses between the two optical fibers at high altitudes, it is key to consider how to reduce axial offset and angular tilt, especially 
axial offset. What is proven in our study is that within the loss budget, MFD mismatch and end face spacing can be ignored in the altitude environment. With the altitude of more than $3000 \mathrm{~m}$, the splice loss increases significantly and can be reduced by properly adjusting the configuration of fusion parameters at this time. However, when the altitude is more than $5200 \mathrm{~m}$, it is almost difficult to successfully splice under general conditions. In order to achieve splice successfully, we need to think of other methods, such as improving performance of splice machine and so on. It is worth noting that although our research team is the first to study the splice loss in high altitude environment, we have successfully identified the main causes of high splice loss in high altitude environment, as well as the approach to achieve fusion-splicing successfully and the method to reduce splice loss as much as possible. The splice loss of most fusion points achieved successfully has been reduced by at least $0.07 \mathrm{~dB}$. Ultimately, the method presented has been directly applied to the splicing engineering in the high altitude environment, achieving good results, greatly saving time cost and economic cost of constructing long-span and super-long-distance optical fiber communication link in high altitude area, which has certain implications for future research of splicing loss in the high altitude environment. Besides, the experimental data obtained from field experiments in the high altitude environment have expanded data reserve of splice loss data library.

Author Contributions: Supervision, C.Y.; writing—original draft preparation, L.H. Both authors have read and agreed to the published version of the manuscript.

Funding: This work is supported by the Science and Technology Project of State Grid Corporation of China (SGLNDK00KJJS1700200) and the National Key R\&D Program of China (2018YFB1800800, 2018YFB1800805, 2020YFB1807900).

Institutional Review Board Statement: Not applicable.

Informed Consent Statement: Not applicable.

Data Availability Statement: Not applicable.

Acknowledgments: The authors would like to thank the editor and the reviewers for their reviews and suggestions to help us improve the quality of this paper.

Conflicts of Interest: The authors declare no conflict of interest.

\section{References}

1. Ghatak, A.; Thyagarajan, K. Introduction to Fiber Optics: Basic Optics; Cambridge University Press: Cambridge, UK, 1998.

2. Das, A.K.; Bhattacharyya, S. Optimum conditions for fusion splicing of optical fiber. Proc. IEEE 1984, 72, 983-984. [CrossRef]

3. Yablon, A.D. Optical Fiber Fusion Splicing; Springer: Berlin/Heidelberg, Germany, 2005.

4. Krause, J.T.; Reed, W.A.; Walker, K.L. Splice loss of single-mode fiber as related to fusion time, temperature, and index profile alteration. J. Light. Technol. 2003, 4, 837-840. [CrossRef]

5. Mohanna, Y. Electric arc temperature estimation of a fibre splicer. IEE Proc. Optoelectron. 2002, 142, 313-315. [CrossRef]

6. Ratuszek, M.; Zakrzewski, Z.; Majewski, J.; Strozecki, S.; Kula, W. Influence of weather conditions on splicing process and parameters of splicing single-mode telecommunication fibers of different types. Proc. SPIE 1999, 3731, 128-132.

7. Ratuszek, M.; Zakrzewski, Z.; Majewski, J.; Strozecki, S.; Zalewski, J. Arc fusion splicing of telecommunication optical fibers in extreme climatic circumstances. In Lightguides and their Applications; International Society for Optics and Photonics: Krasnobrod, Poland, 2000.

8. Hatakeyama, I.; Tsuchiya, H. Fusion splices for single-mode optical fibers. IEEE J. Quantum Electron. 1978, 67, 614-619. [CrossRef]

9. Das, A.; Bhattacharyya, S. Low-loss fusion splices of optical fibers. J. Light. Technol. 1985, 3, 83-92. [CrossRef]

10. Das, A.K.; Mukhopadhyay, A.; Paul, A.; Paul, D.K. Optimum fusion splicing condition of optical fibers. Proc. SPIE 1990, 1174, 358-368.

11. Kato, Y.; Seikai, S.; Tateda, M. Arc-fusion splicing of single-mode fibers. 1: Optimum splice conditions. Appl. Opt. 1982, 21, 1332-1336. [CrossRef] [PubMed]

12. Long, C.W. Modeling of Glass Flow During Arc Fusion Splicing of Fiber Optic Filaments; Dissertation Abstracts International; The University of Tennessee: Knoxville, TN, USA, 1989; Volume 50, Section B, p. 5262.

13. Marcuse, D. Loss Analysis of Single-Mode Fiber Splices. Bell Syst. Tech. J. 1977, 56, 703-718. [CrossRef]

14. Neumann, E.G. Single-Mode Fibers: Fundamentals; Springer: Berlin/Heidelberg, Germany, 1988; Volume 57, pp. $201-203$. 
15. Blanchard, R.L, A new algorithm for computing inertial altitude and vertical velocity. IEEE Trans. Aerosp. Electron. Syst. 1971, 7, 1143-1146. [CrossRef]

16. Xiang, H.; Chen, B.; Yang, M.; Li, C. Altitude measurement based on characteristics reversal by deep neural network for VHF radar. IET Radar Sonar Navig. 2019, 13, 98-103. [CrossRef]

17. Thapa, R.; Gattass, R.R.; Nguyen, V.; Chin, G.; Gibson, D.; Kim, W.; Shaw, L.B.; Sanghera, J.S. Low-loss, robust fusion splicing of silica to chalcogenide fiber for integrated mid-infrared laser technology development. Opt. Lett. 2015, 40, 5074-5077. [CrossRef] [PubMed]

18. Kihara, M.; Tomita, S.; Haibara, T. Influence of wavelength and temperature changes on optical performance of fiber joints with small gap. IEEE Photonics Technol. Lett. 2006, 18, 2120-2122. [CrossRef]

19. Eguchi, M.; Tsuji, Y. Influence of reflected radiation waves caused by large mode field and large refractive index mismatches on splice loss evaluation between elliptical-hole lattice core holey fibers and conventional fibers. J. Opt. Soc. Am. B 2013, 30, 410-420. [CrossRef]

20. Parmar, V.; Bhatnagar, R.; Kapur, P. Optimized butt coupling between single mode fiber and hollow-core photonic crystal fiber. Opt. Fiber Technol. 2013, 19, 490-494. [CrossRef]

21. Kato, Y.; Seikai, S.; Shibata, N.; Tachigami, S.; Watanabe, O. Arc-fusion splicing of single-mode fibers. 2: A practical splice machine. Appl. Opt. 1982, 21, 1916-1921. [CrossRef]

22. Zheng, W. Real time control of arc fusion for optical fiber splicing. J. Light. Technol. 1993, 11, 548-553. [CrossRef]

23. Hatakeyama, I.; Tsuchiya, H. Fusion splices for optical fibers by discharge heating. Appl. Opt. 1978, 17, 1959-1964. [CrossRef]

24. Werzinger, S.; Bunge, C.A. Statistical analysis of intrinsic and extrinsic coupling losses for step-index polymer optical fibers. Opt. Express 2015, 23, 22318-22329. [CrossRef] [PubMed]

25. Nichols, J.M.; Michalowicz, J.V.; Bucholtz, F. Distribution of splice loss in single mode optical fiber. Appl. Opt. 2018, 57, 1140-1150. [CrossRef] [PubMed]

26. Cui, Z.; Yuan, C. Modeling the splice loss of single-mode optical fibers affected by altitude. IEEE Access 2019, 1, 99. [CrossRef]

27. Tamura, Y. Ultra-Low Loss Silica Core Fiber; CLEO-Science and Innovations: San Diego, CA, USA, 2018.

28. Liu, F.L. Detection and Estimation of Connection Splice Events in Fiber Optics Given Noisy OTDR Data; Queen's University: Kingston, ON, Canada, 1998.

29. Kawata, O.; Hoshino, K.; Miyajima, Y.; Ohnishi, M.; Ishihara, K. A splicing and inspection technique for single-mode fibers using direct core monitoring. J. Light. Technol. 1984, 2, 185-191. [CrossRef] 